\title{
Mapping improvement options of government services: illustration of 'concept-mapping'*
}

\author{
Gyula Vastag \\ Professor \\ Széchenyi István University, \\ National University of Public \\ Service, \\ Hungary \\ E-mail: vastag.gyula@sze.hu
}

Ágnes Jenei
Associate Professor
National University of Public
Service,
Hungary
E-mail: jenei.agnes@uni-nke.hu

This paper introduces concept mapping - one form of 'structured conceptualization' - through describing and structuring improvement options of Hungarian government service centres (in Hungarian, these service centres are also known as 'government windows' or 'kormányablakok'). Hungarian service centres are implementations of the 'one-stop shop' concept where almost all government service needs (close to 2,500 cases or procedures; the most common ones are about ID cards, passports and vehicle registrations) of citizens can be met independently of their residence. Consequently, successful implementation of this concept requires new, service-oriented attitude from the staff and much higher level of technical integration than before. Concept mapping uses inputs from stakeholders (managers and staff members of these centres) to describe the problem domain (service improvement options in this example), using natural language input (Hungarian in this case). These inputs are then grouped (by multi-dimensional scaling and Ward hierarchical clustering) and rated by importance. Inputs from the stakeholders were solicited continually throughout the project to determine the final number of clusters and to develop an action plan for improvement, considering the relative importance of the options. Pattern matching was also used to highlight differences between stakeholder groups.

\section{KEYWORDS:}

Service improvement.

Public administration.

Concept mapping.

DOI: $10.35618 / \mathrm{hsr} 2019.01 . e n 024$

* This research was supported by the National University of Public Service under the KÖFOP-2.1.2VEKOP-15-2016-00001 priority project entitled 'Public Service Development Establishing Good Governance' in the 'Servitization of Public Services' Ludovika Research Group. 
Concept mapping is a 'structured conceptualization' methodology (TrochimLinton [1986]; Trochim [1989a], [1989b], [1989c]; Trochim [1990]; Chen-Rossi [1983], [1987]; Trochim-Cook-Setze [1994]). To this day, this approach is rarely used outside the field of program design and control; papers by Rondinelli and Vastag [2000] and Vastag and Melnyk [2002] examined the impacts of the ISO 14001 environmental management system with the approach described here. Concept mapping can also be used for organisational development, as we illustrate in this paper. This inductive, bottom-up approach is based on the active participation of a team that is aware of all aspects of a problematic situation and can represent them. The essence of the methodology is to ask the team members about the options that can lead to the improvement of the situation or about the changes that have occurred as a result of a particular program. The ideas they put forward are represented in two-dimensional maps, indicating the importance of ideas and the potential contradictions among team members. A facilitator guides the process but his or her role is only to manage the process - the content, interpretation and utilization of the concept maps are determined entirely by the group or organisation. Concept mapping is ideal for groups and organisations, because it is: 1. participant-oriented - allowing all of the participants to have a say on the final product, the service production processes, or the delivery of services; 2 . inductive (bottom-up) - starting with everyday concrete ideas that people are familiar with and moving gradually to more general abstract ideas; 3 . structured - with a definite beginning, middle, and end that prevents endless meetings or discussions; 4. simple and intuitive - requiring that participants be able to brainstorm, sort, and rate, all fairly common and familiar activities.

Concept mapping gives easily interpretable results. In this approach, ideas are represented in the form of a picture or map. To construct the map, ideas first have to be described or generated, and the interrelationships among them articulated. Multidimensional scaling and cluster analysis are then applied to this information and the results are depicted in map form. The content of the map is entirely determined by the participants, researchers are facilitating the process. Participants brainstorm the initial ideas, provide information about how these ideas are related, interpret the results of the analysis, and later decide how the map is to be utilised.

In this approach, validity refers to the best available approximation of the truth of propositions (Cook-Campbell [1979]) and the trustworthiness of the methods and results (Guba-Lincoln [1981]). Dumont [1989] examined whether the statistical analysis in concept mapping, in the spirit of construct validity, is doing what it purports to do; her findings supported the validity of map analysis. 
The concept mapping process consists of six steps. The first preparatory stage includes selecting the participants and developing the focus or domain for the conceptualization. Once the participants and the focus have been defined, the second step generates statements that ideally should represent the entire conceptual domain for the topic of interest. In the third step, the participants provide information about how the statements are related to each other. In step four, the conceptual domain is represented graphically. Several interrelated maps, like different views of the same structure, are produced. Step five involves interpreting the maps while the last step focuses on the utilization of the maps.

Most of the concept mapping can be done online or it can be accomplished in two meetings. After selecting the unit of analysis and the participants go through some required steps of introductions and assurances (no right and wrong answers, no publication without their approval), the participants are asked to: "Generate statements that describe the ways of improving of the services we, this particular unit, are providing. These statements are short sentences that reflect your observations. Perhaps, it may be easier to write these statements if you start with the following prompt: 'One specific way to improve our services is to ...' The objective is to list as many statements as you can think of. Each observation, however insignificant it may seem, is important and counts." Statement generation can be done by emailing each participant separately and collecting his/her suggestions or it can be done in a group meeting.

\section{Mapping improvement options of Hungarian government services}

In this paper, we give a step-by-step overview of the concept mapping method and the whole process. In order to do that, we are going to use the suggestions on how to develop services offered by 'government windows' (called 'kormányablakok') in Hungary. These suggestions were generated on site by seven civil servant participants of a customer service skills training in September 2018. All civil servants worked for various Hungarian government windows but for different ones. The facilitator of the training was one of the authors, Agnes Jenei. The training was part of the continuing education program specifically designed for government window staff. Although these seven civil servants participated together in a two-day training program, they did not work together at the same government window. They formed a mixed group, representing several government windows operating in different parts of Hungary. 
As civil servants did not come from the same government window, results are used for illustrating the potential of the methodology, and not for the development of one specific government window. However, these results can give insights into the challenges and problems the government windows are facing and into the opportunities for further development of the one-stop shop concept in Hungary (Ongaro [2004]).

During the above-mentioned training, in one session, participants were asked by the facilitator to describe their opinions, suggestions, ways through which services could be further developed. The following 'prompt' helped them to start thinking and formulating suggestions: "In order to develop government windows' services, I would suggest the following ..." This opening sentence was completed by the seven participants. Each participant wrote down 5-10 statements. After they finished, the facilitator collected these lists and displayed all statements on a flipchart. The statements (suggestions) generated in this way were checked grammatically, and the overlaps were eliminated. Participants were asked to re-check and complete the list of suggestions (statements). At this point, they could modify and expand the list. When all statements were on the list, apart from the obvious mistakes (spelling and grammar, for example) it was important not to change the suggestions in any other way. Finally, 35 suggestions (statements) remained on the list that all participants agreed with. These 35 suggestions (statements) covered the entire area of potential service development, according to the participants, familiar with all aspects of government windows.

In the next step, statements thus obtained were assigned a random number between 1 and 35 for identification and then were sorted by this number. The resulting random order of the 35 statements is shown in the Appendix.

After finalizing the problem domain, each of the seven participants was given a complete 'card package': 35 cards with the statements and identification numbers. Each participant was asked to sort these cards into piles - following the instructions of Rosenberg and Kim [1975], Weller and Romney [1988] on grouping unstructured cards - in a way that made sense to them. There were two sorting restrictions: each card could be placed in one pile only and the number of piles had to be more than one and less than 35 .

After sorting, participants were asked to rate the statements: "Please rate each statement on a five-point scale in terms of how important you think the statement is for the government window's service development, where $1=$ relatively unimportant (compared with the rest of the statements), $2=$ somewhat important, $3=$ moderately important, $4=$ very important, $5=$ extremely important. Please keep in mind that none of these 35 statements are totally unimportant, so this rating should be considered a relative judgement of the importance of each item to all other statements."

Based on the sorting results, for each participant a binary symmetric matrix of similarities was formed; $X_{i j}^{k}=1$ if statements $i$ and $j$ were placed in the same pile by par- 
ticipant $k, 0$ otherwise $(i, j=1,2, \ldots, 35 ; k=1,2, \ldots, 7)$. Aggregating the individual matrices of similarities, the total (or group) similarity matrix was computed. The total similarity matrix was obtained as the sum of individual similarity matrixes. In this $35 \times 35$ symmetric matrix, the value of the cell $(i, j)$ was the number of participants who put the $i$ and $j$ suggestions into one group. The higher this value was, the more similar the participants thought the two statements were. The diagonal of the matrix, by definition, was filled with 7-s (the number of participants).

The total similarity matrix served the input to a two-dimensional nonmetric MDS (multi-dimensional scaling). MDS placed those suggestions (statements) closer to each other in the two-dimensional space that were piled together by more participants. It is generally easier and more comfortable to work with two-dimensional configurations, as results in the two-dimensional space are much easier to understand and interpret than in higher dimensions (Kruskal-Wish [1978] p. 58.).

'Stress' value is the commonly used statistic to measure the goodness of fit of the two-dimensional configuration to the original similarity matrix. The smaller this value is, the better the two-dimensional representation would be. Although there are no clear prescriptions or rules on the stress value, Trochim [1993] reported 0.285 as the average stress value across 33 concept mapping projects.

In our case, in the final two-dimensional configuration, the stress value was 0.130 , and this two-dimensional configuration explained $91.1 \%$ of all variances. Calculations and illustrations were made with JMP (SAS) 14.10.

The two-dimensional coordinates of the 35 suggestions for improvement were used as inputs to hierarchical clustering. As choosing a clustering procedure is somewhat subjective (Anderberg [1973]; Everitt [1980]; Fisher-Van Ness [1971]; Milligan [1980], [1981]), we followed Trochim's [1989a] suggestion, who, after trying several methods, recommended using Ward's algorithm. Ward's algorithm instead of single linkage (nearest neighbour principle), full linkage (farthest neighbour principle), or centroid methods, minimises the within-cluster sum of squares to the between-cluster sum of squares at each level of joining.

The number of clusters was determined in several iterative consultations with the group. After examining several clusters, a five-cluster solution was selected. (See Figure 1.) The numbers correspond to the statement identifiers listed in the Appendix. For example, suggestion 1 with the approximate coordinates of $(-0.5,-1.0)$ in Cluster 1 , stands for "I would abolish the hiring freeze of staff members. I would increase the number of the staff members in the government window and fill the empty counters, as the clerks are often overburdened, stressed, and tense." Also, a random, slight variation ('jittering') was added to the co-ordinates to avoid masking that is the overlap of two points. 
Figure 1. Suggestions for development clustered on a two-dimensional map

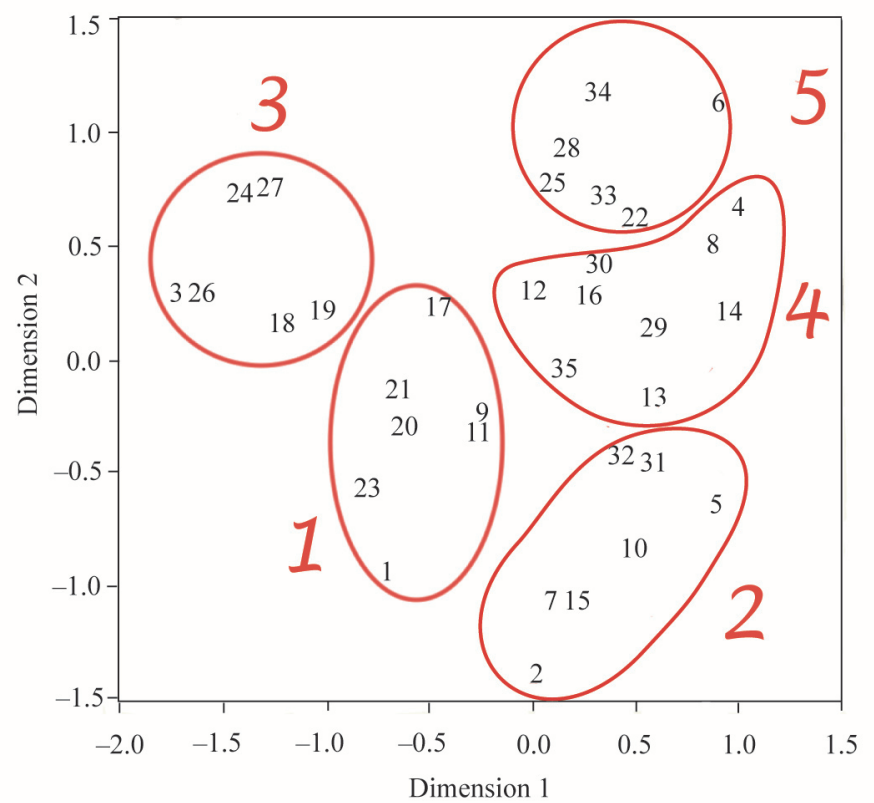

Note. Here and in Figure 2, the numbers correspond to the statement identifiers. (See the Appendix.)

Figure 2. Average importance ratings of suggestions by cluster

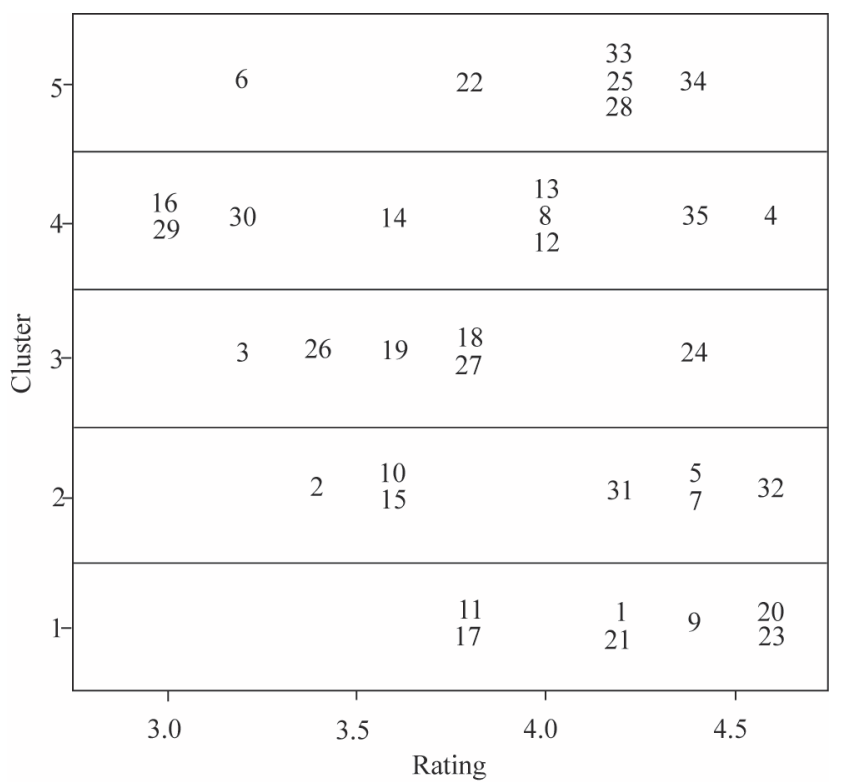


Figure 2 shows the average importance (by the participants) of the suggestions by cluster and the distribution of the average ratings in each cluster. For example, in Cluster 4 two suggestions are tied and were considered less important by the participants: number 16 ("I would dedicate each week a day or a part of a day only to telephone calls.") and number 29 ("I would tell the clients that government workers are also entitled to a lunch break, and I would emphasise our appreciation for their patience and understanding."). These suggestions had an average value of just over 3.0.

Clusters were named based on suggestions in each cluster. The clusters, their given names and the list of suggestions are summarised as follows.

\section{Cluster 1: Management issues}

1 - I would abolish the hiring freeze of staff members. I would increase the number of the staff members in the government window and fill the empty counters, as the clerks are often overburdened, stressed, and tense.

9 - Where no back-office room is available for workers, it would be necessary to redesign the entire government window and create such a room. Back-office work requires intense concentration and a high degree of precision. If there is no separation between the front office and the back-office rooms, then staff members should do back-office work in the presence of clients and cannot work efficiently.

11 - I would recommend that the front office workers should stop working at the front office desks 15-20 minutes before the customer service office closes, as it takes 15-20 minutes to shut down the government window procedures. If clients stay until the closing hour, workers need extra time (15-20 minutes) each day to finish such procedures.

17 - I would give all 'general' clerks of the government window a chance to observe the work of their front office colleagues in specialised (pension, health, family care, construction, etc.) departments. Observing front office work (for two days in each department) would give them the opportunity to learn how to handle more specialized cases of citizens.

20 - I would pressure managers to introduce obligatory lunch breaks for staff members every day even if the front office is overcrowded, as it is important for them to have their daily lunch under calm circumstances.

21 - I would introduce a rest break rotation among clerks to give them the break time provided by law. It is important to allow them to stand up and meet their needs.

23 - Under no circumstances should the managers criticise staff members in public. Criticizing a staff member in front of his/her colleagues is humiliating and if it happens in front of the clients, it reduces the workers' credibility. 


\section{Cluster 2: Process development}

2 - I would not maximise (regulate in advance) the number of clerks that can be placed in a particular performance category. (For example, if everyone is considered excellent, it would be important to be able to place all of them in the 'Excellent' category.)

5 - It would be important not to throw away customer satisfaction questionnaires even if they contain complaints, criticism, or claims. They should be used for service development. I would let clients know of the government window staff's concern towards their complaints or suggestions and show transparently the continuous development in order to improve services and meet their demands.

7 - I would have managers (mid-level leaders) to forward problems and suggestions up the chain.

10 - Currently, telephone calls with clients are not counted as part of workload. It would be necessary to include such calls in daily reports. Civil servants spend a long time with clients on phone, it is part of their work. (For example, 8 clients $\times 15$ minutes $=120$ minutes per working day.)

15 - It would be important to introduce a 'suggestion box' for employees. The head of the government window should open the box in public and analyse all suggestions together with the employees. Good ideas, suggestions and solutions should be reported to higher administrative levels and implemented later.

31 - A clerk's work should only be assessed by a manager who is familiar with it.

32 - I would suggest that the management introduce a continuous improvement process: identify operational problems and then seek solutions to them. I would have management involve workers in identifying and resolving problems. I would encourage staff members to make useful suggestions and reward them for doing so. My advice is to make managers forward problems, suggestions up the chain to find solutions to them. It would be important to reprimand the leadership if they do not do so.

\section{Cluster 3: Security issues and conflict management}

3 - I suggest hiring a security service to protect vulnerable, defenseless government workers in case of physical threats and atrocity.

18 - It would be important for the government window staff to be able to consult regularly (weekly or biweekly) with an external trainer or an expert in conflict management. During consultations, the head of the government window should be present as well.

19 - I would give staff members the opportunity to consult a psychologist once a month (to let the steam off and to prevent psychosomatic illnesses or burnout). 
24 - I would hire a security guard in order to stop clients from entering the office in inappropriate clothing or under the influence of alcohol or drugs.

26 - I suggest the employment of security guards. They could give a lift to the government workers seriously threatened by clients.

27 - In order to protect workers of the government window, I would hire a security guard to stop people under influence of drugs or alcohol from entering the customer service office.

\section{Cluster 4: Information system, technical support}

4 - It would be necessary to have an 'information /reception desk' where the receptionist (not a doorman or a security guard, but a civil servant specialised in the customer service of government windows) could deliver the clients all the necessary information on filling forms, attaching documents, powers of attorney, writing requests, etc. for the issues they would like to arrange. This way, waiting times could be reduced: clients that were not fully prepared to arrange their administrative cases (issues) would go home and return another time with all the necessary documents.

8 - When the card payment system or the computer system is out of order, it would be important to inform customers in the queue through the loudspeaker and signs about the inconvenience, otherwise they might become unsatisfied with the work of the clerks. I suggest establishing a protocol for the warning procedure.

12 - I would provide all technical means for the job (pens, special printer for printing social security cards, etc.). Although government services are done electronically, in many cases, there is a need for a normal pen.

13 - For each type of case, I would set the so-called 'standard time' carefully. Clerks often have to deal with clients having delicate situations. Government workers should communicate with different types of clients, be careful with the language they use, and handle situations in which extreme emotions (anger, sadness, etc.) are present.

14 - I would provide more space between the customer desks to guarantee the privacy of the clients. Currently, everyone can hear others' sensitive data by sitting next to them.

16 - I would dedicate each week a day or a part of a day only to telephone calls.

29 - I would tell the clients that government workers are also entitled to a lunch break, and I would emphasise our appreciation for their patience and understanding.

30 - The last customer should take a number maximum 30 minutes before the end of the office hours.

35 - I propose a weekly meeting where the management of the government window and all the clerks are present. The meeting should deal with the latest legislative and other changes, announcements, challenges, problems; this would be the right time to share good practices, to answer the questions of the workers, to discuss diffi- 
cult cases, and to talk about the behaviour of clients. The meeting should be held during working hours, giving every worker the opportunity to be present.

\section{Cluster 5: Operational issues, scheduling}

6 - The customer call system should provide true information on how many customers are waiting at a given counter (taking into account that the clerk is dealing with more than one type of cases).

22 - If it would shorten waiting times, I would put a photo booth in the government window (even if "it would mean a deviation from the standardized layout and image of the government windows").

25 - It would be necessary to update the 'Information/Knowledge Database' with the latest norms and forms to be filled, otherwise it is useless. It would be vital to set up a relevant protocol between the government window (customer service department) and the specialised departments.

28 - I would re-think, re-design the vocabulary of the Knowledge/Information Database. In many cases, it is different from the language used by the clients. It would be important to give the staff members the opportunity to search keywords featuring the terms preferred by clients.

33 - In order to develop the one-stop shop system, I would take the high complexity of the Hungarian legal system into account: I would not try to adopt the original onestop shop system (fully integrated customer services); instead, I would implement the all services 'under one roof'. Basically, I would bring all the service providers (including specialized services) in one building.

34 - I would improve the interoperability between the special service (health care, family care, etc.) providers' IT systems.

\section{Utilizing the results: action proposals and pattern matching}

Based on the results of concept mapping, an action plan could be formulated. In our illustrative example, priority should be given to the development of managerial/leadership competencies and attitudes, to the introduction of process development measures, and to the elimination of problems hindering operations.

If the project had been carried out in one specific government window, commitment packages (see Friend-Hickling [2005]) could be developed. A commitment package is an action proposal that defines what actions must be taken immediately, or if more exploration is necessary based on time and uncertainty of the decision 
areas. The package also leaves future decision space for deferred choices and contingency planning if there is still enough time to analyse further choices.

It is also important to recognise and address group differences. It is quite common that members of the stakeholder group do not view these suggestions the same way and group members may have characteristics along which these views about importance ratings are distinct. In this case study, we contrasted the importance ratings by gender (male/female). As one of the seven participants did not participate in the rating, the remaining six members were evenly divided between men and women.

Figure 3 shows pattern matching, the average importance ratings men and women assigned to each cluster. While the most important cluster for men was 5 (Operational issues, scheduling), with an average weight of 4.278, for women, Cluster 1 (Management issues) was the most important with an average weight of 4.429. Although there was a very strong correlation $(r=0.872)$ between the importance ratings of the two subgroups (men and women), there were also major differences in order. These differences are well illustrated by the ladder graph of Figure 3.

Figure 3. Differences in average importance ratings by gender

Gender

Average importance

4.278

4.143

4.095

3.722

3.519

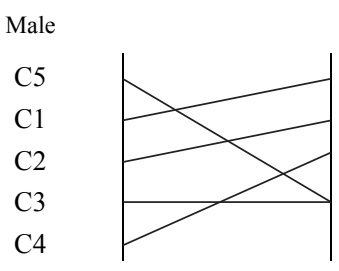

$r=0.872$
Female

C1

$\mathrm{C} 2$

$\mathrm{C} 4$

C3, C5
Average importance

4.429

4.143

4.074

3.889

In case of a perfect match, each rung of the ladder between the rails is horizontal, parallel, not intersecting. This is not the case here, however, and this clearly shows that women's and men's perception patterns, especially regarding Cluster 5 (Operational issues, scheduling), differ significantly: it is the most important item with men and last on the importance list of women. In an action proposal, these differences also have to be considered.

\section{Conclusion}

The paper's contribution is twofold: firstly, it offers a tool, a methodology that is ideally suited for situations where a group, as a whole, is put in charge of an im- 
provement project. It is important to know that the group members - taken together know and can describe all aspects of the problem domain. The methodology, concept mapping, is very much structured, visually appealing and it lends itself to accountability and measurable action proposals.

Secondly, we offer some insights into the changing nature of Hungarian government services that affect almost all aspects of our lives. As we can see from the list of improvement options, the suggested changes involve a wide range of actions and embody both changes in managerial and staff attitudes and major investments in infrastructure and computerization that are very much in line with the global changes in public administration. Perhaps, it is fitting to remember the wildly popular British music hall song from 1912 where the homesick Irish Paddy $O$ in London sang to his sweetheart, Molly $O$, back in Ireland "It's a long way to Tipperary, It's a long, long way to go." Most certainly, public service improvements have been long in the making and, in the kaizen spirit it is still a long way to go. However, on the upside, there has been an undeniable and measurable progress that we all benefitted from.

\section{Appendix}

1 - I would abolish the hiring freeze of staff members. I would increase the number of the staff members in the government window and fill the empty counters, as the clerks are often overburdened, stressed, and tense.

2 - I would not maximise (regulate in advance) the number of clerks that can be placed in a particular performance category. (For example, if everyone is considered excellent, it would be important to be able to place all of them in the 'Excellent' category.)

3 - I suggest hiring a security service to protect vulnerable, defenseless government workers in case of physical threats and atrocity.

4 - It would be necessary to have an 'information /reception desk' where the receptionist (not a doorman or a security guard, but a civil servant specialised in the customer service of government windows) could deliver the clients all the necessary information on filling forms, attaching documents, powers of attorney, writing requests, etc. for the issues they would like to arrange. This way, waiting times could be reduced: clients that were not fully prepared to arrange their administrative cases (issues) would go home and return another time with all the necessary documents.

5 - It would be important not to throw away customer satisfaction questionnaires even if they contain complaints, criticism, or claims. They should be used for service development. I would let clients know of the government window staff's concern towards their complaints or suggestions and show transparently the continuous development in order to improve services and meet their demands. 
6 - The customer call system should provide true information on how many customers are waiting at a given counter (taking into account that the clerk is dealing with more than one type of cases).

7 - I would have managers (mid-level leaders) to forward problems and suggestions up the chain.

8 - When the card payment system or the computer system is out of order, it would be important to inform customers in the queue through the loudspeaker and signs about the inconvenience, otherwise they might become unsatisfied with the work of the clerks. I suggest establishing a protocol for the warning procedure.

9 - Where no back-office room is available for workers, it would be necessary to redesign the entire government window and create such a room. Back-office work requires intense concentration and a high degree of precision. If there is no separation between the front office and the back-office rooms, then staff members should do back-office work in the presence of clients and cannot work efficiently.

10 - Currently, telephone calls with clients are not counted as part of workload. It would be necessary to include such calls in daily reports. Civil servants spend a long time with clients on phone, it is part of their work. (For example, 8 clients $\times 15$ minutes $=120$ minutes per working day.)

11 - I would recommend that the front office workers should stop working at the front office desks 15-20 minutes before the customer service office closes, as it takes 15-20 minutes to shut down the government window procedures. If clients stay until the closing hour, workers need extra time (15-20 minutes) each day to finish such procedures.

12 - I would provide all technical means for the job (pens, special printer for printing social security cards, etc.). Although government services are done electronically, in many cases, there is a need for a normal pen.

13 - For each type of case, I would set the so-called 'standard time' carefully. Clerks often have to deal with clients having delicate situations. Government workers should communicate with different types of clients, be careful with the language they use, and handle situations in which extreme emotions (anger, sadness, etc.) are present.

14 - I would provide more space between the customer desks to guarantee the privacy of the clients. Currently, everyone can hear others' sensitive data by sitting next to them.

15 - It would be important to introduce a 'suggestion box' for employees. The head of the government window should open the box in public and analyse all suggestions together with the employees. Good ideas, suggestions and solutions should be reported to higher administrative levels and implemented later.

16 - I would dedicate each week a day or a part of a day only to telephone calls.

17 - I would give all 'general' clerks of the government window a chance to observe the work of their front office colleagues in specialised (pension, health, family 
care, construction, etc.) departments. Observing front office work (for two days in each department) would give them the opportunity to learn how to handle more specialized cases of citizens.

18 - It would be important for the government window staff to be able to consult regularly (weekly or biweekly) with an external trainer or an expert in conflict management. During consultations, the head of the government window should be present as well.

19 - I would give staff members the opportunity to consult a psychologist once a month (to let the steam off and to prevent psychosomatic illnesses or burnout).

20 - I would pressure managers to introduce obligatory lunch breaks for staff members every day even if the front office is overcrowded, as it is important for them to have their daily lunch under calm circumstances.

21 - I would introduce a rest break rotation among clerks to give them the break time provided by law. It is important to allow them to stand up and meet their needs.

22 - If it would shorten waiting times, I would put a photo booth in the government window (even if "it would mean a deviation from the standardized layout and image of the government windows").

23 - Under no circumstances should the managers criticise staff members in public. Criticizing a staff member in front of his/her colleagues is humiliating and if it happens in front of the clients, it reduces the workers' credibility.

24 - I would hire a security guard in order to stop clients from entering the office in inappropriate clothing or under the influence of alcohol or drugs.

25 - It would be necessary to update the 'Information/Knowledge Database' with the latest norms and forms to be filled, otherwise it is useless. It would be vital to set up a relevant protocol between the government window (customer service department) and the specialised departments.

26 - I suggest the employment of security guards. They could give a lift to the government workers seriously threatened by clients.

27 - In order to protect workers of the government window, I would hire a security guard to stop people under influence of drugs or alcohol from entering the customer service office.

28 - I would re-think, re-design the vocabulary of the Knowledge/Information Database. In many cases, it is different from the language used by the clients. It would be important to give the staff members the opportunity to search keywords featuring the terms preferred by clients.

29 - I would tell the clients that government workers are also entitled to a lunch break, and I would emphasise our appreciation for their patience and understanding.

30 - The last customer should take a number maximum 30 minutes before the end of the office hours. 
31 - A clerk's work should only be assessed by a manager who is familiar with it.

32 - I would suggest that the management introduce a continuous improvement process: identify operational problems and then seek solutions to them. I would have management involve workers in identifying and resolving problems. I would encourage staff members to make useful suggestions and reward them for doing so. My advice is to make managers forward problems, suggestions up the chain to find solutions to them. It would be important to reprimand the leadership if they do not do so.

33 - In order to develop the one-stop shop system, I would take the high complexity of the Hungarian legal system into account: I would not try to adopt the original onestop shop system (fully integrated customer services); instead, I would implement the all services 'under one roof'. Basically, I would bring all the service providers (including specialized services) in one building.

34 - I would improve the interoperability between the special service (health care, family care, etc.) providers' IT systems.

35 - I propose a weekly meeting where the management of the government window and all the clerks are present. The meeting should deal with the latest legislative and other changes, announcements, challenges, problems; this would be the right time to share good practices, to answer the questions of the workers, to discuss difficult cases, and to talk about the behaviour of clients. The meeting should be held during working hours, giving every worker the opportunity to be present.

\section{References}

Anderberg, M. R. [1973]: Cluster Analysis for Applications. Academic Press. New York.

CHEN, H.-T. - Rossi, P. H. [1983]: Evaluating with sense: the theory-driven approach. Evaluation Review. Vol. 7. Issue 3. pp. 283-302. https://doi.org/10.1177/0193841X8300700301

CHEN, H.-T. - Rossi, P. H. [1987]: The theory-driven approach to validity. Evaluation and Program Planning. Vol. 10. Issue 1. pp. 95-103. https://doi.org/10.1016/0149-7189(87)90025-5

CoOK, T. D. - CAmpBell, D. T. [1979]: Quasi-experimentation: Design and Analysis Issues for Field Settings. Rand McNally. Chicago.

DumONT, J. [1989]: Validity of multidimensional scaling in the context of structured conceptualization. Evaluation and Program Planning. Vol. 12. Issue 1. pp. 81-86. https://doi.org/10.1016/0149-7189(89)90026-8

EveritT, B. [1980]: Cluster Analysis. $2^{\text {nd }}$ Edition. John Wiley \& Sons Inc. New York.

Fisher, L. - VAN NeSS, J. W. [1971]: Admissible clustering procedures. Biometrika. Vol. 58. Issue 1. pp. 91-104. https://doi.org/10.1093/biomet/58.1.91

Friend, J. - Hickling, A. [2005]: Planning Under Pressure: The Strategic Choice Approach. $3^{\text {rd }}$ Edition. Urban and Regional Planning Series. Elsevier Butterworth-Heinemann. Oxford.

GubA, E. G. - Lincoln, Y. S. [1981]: Effective Evaluation. Jossey-Bass. San Francisco. 
Kruskal, J. B. - Wish, M. [1978]: Multidimensional Scaling. Sage University Paper Series on Quantitative Applications in the Social Sciences. No. 07-011. Sage Publications. Newbury Park. http://dx.doi.org/10.4135/9781412985130

MilligAN, G. W. [1980]: An examination of the effect of six types of error perturbation on fifteen clustering algorithms. Psychometrika. Vol. 45. No. 3. pp. 325-342. http://dx.doi.org/ 10.1007/BF02293907

Milligan, G. W. [1981]: A review of Monte Carlo tests of cluster analysis. Multivariate Behavioral Research. Vol. 16. Issue 3. pp. 379-407. https://doi.org/10.1207/s15327906mbr1603_7

Ongaro, E. [2004]: Process management in the public sector - The experience of one-stop shops in Italy. The International Journal of Public Sector Management. Vol. 17. No. 1. pp. 81-107. http://dx.doi.org/10.1108/09513550410515592

Rondinelli, D. A. - Vastag, G. [2000]: Panacea, common sense, or just a label? The value of ISO 14001 Environmental management systems. European Management Journal. Vol. 18. No. 5. pp. 499-510. http://dx.doi.org/10.1016/S0263-2373(00)00039-6

RosenberG, S. - KIM, M. P. [1975]: The method of sorting as a data-gathering procedure in multivariate research. Multivariate Behavioral Research. Vol. 10. No. 4. pp. 489-502. http://dx.doi.org/10.1207/s15327906mbr1004_7

Trochim, W. - CoOK, J. - SETZE, R. [1994]: Using concept mapping to develop a conceptual framework of staff's views of a supported employment program for persons with severe mental illness. Consulting and Clinical Psychology. Vol. 62. No. 4. pp. 766-775. https://doi.org/10.1037//0022-006X.62.4.766

Trochim, W. - Linton, R. [1986]: Conceptualization for evaluation and planning. Evaluation and Program Planning. Vol. 9. Issue 4. pp. 289-308. https://doi.org/10.1016/0149-7189(86)90044-3

Trochim, W. [1985]: Pattern matching, validity, and conceptualization in program evaluation. Evaluation Review. Vol. 5. No. 9. pp. 575-604. https://doi.org/10.1177/0193841X8500900503

Trochim, W. [1989a]: An introduction to concept mapping for planning and evaluation. Evaluation and Program Planning. Vol. 12. No. 12. pp. 1-16. https://doi.org/10.1016/01497189(89)90016-5

Trochim, W. [1989b]: Concept mapping: soft science or hard art? Evaluation and Program Planning. Vol. 12. Issue 1. pp. 87-110. https://doi.org/10.1016/0149-7189(89)90027-X

Trochim, W. [1989c]: Outcome pattern matching and program theory. Evaluation and Program Planning. Vol. 12. Issue 4. pp. 355-366. https://doi.org/10.1016/0149-7189(89)90052-9

Trochim, W. [1993]: Reliability of Concept Mapping. Paper presented at the Annual Conference of the American Evaluation Association. 6 November. Dallas.

VastaG, G. - MelnYK, S. A. [2002]: Certifying environmental management systems by the ISO 14001 standards. International Journal of Production Research. Vol. 40. No. 18. pp. 4743-4763. https://doi.org/10.1080/00207540210161632

Weller, S. C. - Romney, A. K. [1988]: Systematic Data Collection: Qualitative Research Methods. Vol. 10. Sage. Beverly Hills. 